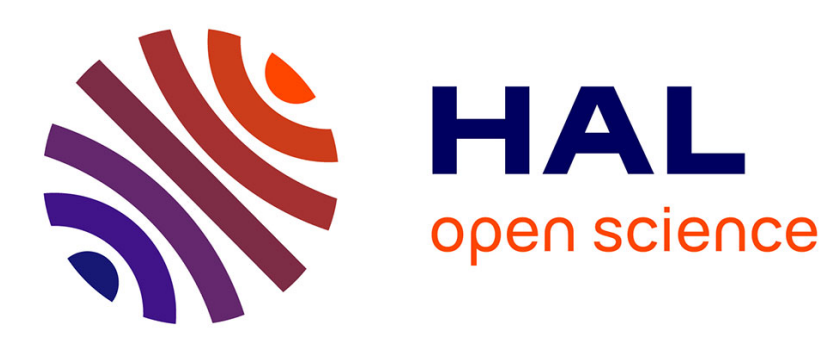

\title{
Anti-mitochondrial antibodies are not a hallmark of severity in idiopathic inflammatory myopathies
}

Wladimir Mauhin, Kuberaka Mariampillai, Yves Allenbach, Jean-Luc

Charuel, Lucile Musset, Olivier Benveniste

\section{- To cite this version:}

Wladimir Mauhin, Kuberaka Mariampillai, Yves Allenbach, Jean-Luc Charuel, Lucile Musset, et al.. Anti-mitochondrial antibodies are not a hallmark of severity in idiopathic inflammatory myopathies. Joint Bone Spine, 2017, 10.1016/j.jbspin.2017.04.004 hal-01518320

\section{HAL Id: hal-01518320 \\ https: / hal.sorbonne-universite.fr/hal-01518320}

Submitted on 4 May 2017

HAL is a multi-disciplinary open access archive for the deposit and dissemination of scientific research documents, whether they are published or not. The documents may come from teaching and research institutions in France or abroad, or from public or private research centers.
L'archive ouverte pluridisciplinaire HAL, est destinée au dépôt et à la diffusion de documents scientifiques de niveau recherche, publiés ou non, émanant des établissements d'enseignement et de recherche français ou étrangers, des laboratoires publics ou privés. 


\section{Anti-mitochondrial antibodies are not a hallmark of severity in idiopathic inflammatory myopathies}

Wladimir Mauhin ${ }^{a}$; Kuberaka Mariampillai ${ }^{a}$; Yves Allenbach ${ }^{a}$; Jean-Luc Charuel ${ }^{b}$; Lucile Musset $^{\text {b }}$; Olivier Benveniste $^{a}$

A : Internal Medicine and Clinical Immunology, Hôpital Pitié-Salpêtrière, UMR 974, UPMC, AP-HP, 75013 Paris, France

B : Immunology Department, Hôpital Pitié-Salpêtrière, AP-HP, 75013 Paris, France

Corresponding author: Olivier Benveniste

Hôpital Pitié-Salpêtrière, Médecine Interne et Immunologie Clinique

47-83, boulevard de l'hôpital, 75013 Paris

Olivier.benveniste@aphp.fr

Phone : $33(0) 142177622 \quad$ Fax : $33(0) 142161058$

Key words:

anti-mitochondrial antibodies, myositis, auto-antibodies, primary biliary cholangitis 
Anti-mitochondrial antibodies type 2 (AMA2) are the hallmarks of primary biliary cholangitis (PBC) [1]. AMA2 have also been described in $11.3 \%$ of idiopathic inflammatory myopathies (IIM) then associated with cardiac involvement, muscular atrophy and granuloma (table 1) [2].

Screening our Myositis database in November 2015, 142 patients had information for AMA2. A muscular lymphoma was excluded. Finally, 11/141 patients (7.8\%) were analyzed as AMA2-positive (AMA2+; age at diagnosis 22.7-76.7y.).Proximal muscular weakness was observed in all but two patients with antisynthetase syndromes. Two patients associated axial involvement, one patient had proximal, axial and distal weakness. Eight AMA2+ patients had difficulties to climb stairs, 6 for walking, 3 for swallowing. Median (interquartile) CK level at diagnosis was 1500U/L (481-8100) in AMA2+ vs 3843 (1050-9000; $\mathrm{p}=0.20)$. Among AMA2+, $8 / 9$ had T2-weighted hypersignals on muscular MRI with atrophy in $3(33.0 \%$ vs 52.9\%; $p=0.19)$. Cardiac involvement was diagnosed in $4 / 11$ AMA2+ versus $36 / 122(p=0.73)$. One patient exhibited biventricular dilatation with reduced left ventricular function (37\%); two others had de novo rhythm troubles and one presented hypokinesia on echography. Cardiac MRI showed hyperT2-weighted signals and gadolinium enhancement in these patients. According to the clinical and pathological ENMC criteria [3], a trend towards higher prevalence of polymyositis was observed in AMA2+ group (5/11 vs 25/130; $p=0.06)$. AMA2+ biopsies revealed no granuloma or particular feature.

AMA2+ patients exhibited less cutaneous manifestations $3 / 11$ vs $79 / 122(p=0.02)$. Specific idiopathic pneumopathy was observed in 3/11 AMA2+ with fibrosis in one, versus 56/108 AMA2- ( $p=0.2)$. Two of these three patients had anti-synthetase syndrome associated with cutaneous manifestation in an antiJo1 patient. Arthralgia and Raynaud phenomenon were not different between groups. Primary biliary cirrhosis was previously diagnosed in 3 AMA2+ patients without significant association with cardiac involvement. Among AMA2+, five had associated auto-immune disease: lupus, Sjögren, antiphospholipid, anti-synthetase syndromes, rheumatoid arthritis and Hashimoto's thyroiditis. Among 
AMA2+, one had hepatitis C virus associated-hepatocarcinoma, another had polycythemia vera. No other myositis-associated antibody was detected in 2 AMA2+ patients whereas others had anti-signal recognition particle $(n=1)$, anti-HMGCoA-reductase $(n=1)$, anti-Ro52 $(n=4)$, anti-PL7 $(n=1)$, anti-Jo1 $(n=1)$. Among AMA2+ with cardiac involvement, 1 had anti-Ro52, another anti-HMGCoA-reductase antibodies.

AMA2+ patients with cardiac involvement received $1 \mathrm{~g}$ methylprednisolone infusions (MP; $n=3$ ) plus intravenous immunoglobulin (IVIg; $n=2$ ), cyclophosphamide (CP; $n=3$ ), mycophenolate mofetil (MMF; $\mathrm{n}=1$ ) and/or plasma-exchanges (EP; $n=2)$, all associated with long-term oral prednisone $(1 \mathrm{mg} / \mathrm{kg} / \mathrm{d}$. then tapered) and methotrexate (MTX; $n=3$ ) or azathioprine (AZA; $n=1$ ). Two received rituximab as secondline treatment. AMA2+ patients without cardiac involvement received oral prednisone $(1 \mathrm{mg} / \mathrm{kg} / \mathrm{d}$.; $n=7 / 7)$, IVIg (n=3), MMF (n=1), EP(n=3), MP (n=3), MTX (n=3), AZA (n=1). One patient died from liver failure, 6 improved muscular strength whereas deficit remained stable in 4.

The limitation of this study is the absence of systematic screening for AMA2 in our whole IIMs population. Prospective studies are needed. Interestingly, no IIM was reported in a prospective study of AMA2+ patients $(n=1318)$ [4]. AMA2 seem not associated with cardiac involvement, muscular atrophy or granuloma, neither with a specific pattern of IIM and not appear as a hallmark of severity.

\section{Acknowledgements:}

No acknowledgement

\section{Conflict of interest statement:}

The authors declare no conflicts of interest

\section{Funding Statement:}

This work was not supported by any funding 


\section{References}

[1] Selmi C, Bowlus CL, Gershwin ME, Coppel RL. Primary biliary cirrhosis. Lancet Lond Engl 2011;377:1600-9.

[2] Maeda MH, Tsuji S, Shimizu J. Inflammatory myopathies associated with anti-mitochondrial antibodies. Brain J Neurol 2012;135:1767-77.

[3] Hoogendijk JE, Amato AA, Lecky BR, Choy EH, Lundberg IE, Rose MR, et al. 119th ENMC international workshop: trial design in adult idiopathic inflammatory myopathies, with the exception of inclusion body myositis, 10-12 October 2003, Naarden, The Netherlands. Neuromuscul Disord NMD 2004;14:337-45.

[4] Dahlqvist G, Gaouar F, Carrat F, Meurisse S, Chazouillères O, Poupon R, et al. Large-scale characterization study of patients with antimitochondrial antibodies but nonestablished primary biliary cholangitis. Hepatol Baltim Md 2017;65:152-63. 
Table 1. Characteristics of Anti-Mitochondrial antibodies associated myositis.

\begin{tabular}{|c|c|c|c|c|}
\hline & \multicolumn{3}{|c|}{ Our cohort } & Myositis database \\
\hline & $\begin{array}{c}\text { AMA2+ } \\
\text { n (\%) }\end{array}$ & $\begin{array}{c}\text { AMA2- } \\
\mathrm{n}(\%)\end{array}$ & $p$ & $\begin{array}{c}\text { all } \\
\text { n (\%) }\end{array}$ \\
\hline$n=$ & $11 / 141(7.8)$ & $130 / 141(92.2)$ & - & 1098 \\
\hline Mean age at onset (y.) & 45.6 & 46 & - & $53(n=492)$ \\
\hline Median CK at diagnosis (U/L) & 1500 & 3843 & 0.20 & $5203.84(n=441)$ \\
\hline Muscle atrophy & $3 / 9(33.0)$ & $36 / 68(52.9)$ & 0.19 & $129 / 226(57.07)$ \\
\hline Primary Biliary Cholangitis & $3 / 11(27.3)$ & nd & $N d$ & ND \\
\hline Cardiac involvement & 4/11 (36.4) & $36 / 122(29.5)$ & 0.73 & $133 / 452(29.42)$ \\
\hline Arthralgia & $7 / 11(63.6)$ & $57 / 119(47.9)$ & 0.36 & $156 / 446(34.97)$ \\
\hline Cutaneous manifestation & $3 / 11(27.3)$ & $79 / 122(64.8)$ & 0.02 & $243 / 500(48.6)$ \\
\hline Raynaud phenomenon & $5 / 11(45.5)$ & $51 / 120(42.5)$ & 1.00 & $118 / 435(27.12)$ \\
\hline Lung involvement & $3 / 11(27.3)$ & $56 / 108(51.8)$ & 0.20 & $143 / 368(38.85)$ \\
\hline Granuloma & $0 / 11(0.0)$ & $2 / 55(3.6)$ & 1.00 & $7 / 194(3.6)$ \\
\hline Anti-synthetase syndrome & $2 / 11(18.2)$ & $26 / 130(20.0)$ & 1.00 & 114/1098 (10.38) \\
\hline Immune Mediated & & & & \\
\hline Necrotizing Myopathy & $2 / 11(18.2)$ & $35 / 130(27.0)$ & 0.73 & $127 / 1098(11.56)$ \\
\hline Dermatomyositis & 1/11 (9.1) & $22 / 130(17.0)$ & 0.69 & $173 / 1098(15.75)$ \\
\hline Polymyositis & $5 / 11(45.5)$ & 25/130 (19.0) & 0.06 & 197/1098 (17.94) \\
\hline Inclusion Body Myositis & $0 / 11(0.0)$ & $17 / 130(13.1)$ & 0.36 & $315 / 1098(28.68)$ \\
\hline Myositis Specific antibodies & $4 / 11(36.4)$ & 74/124 (59.7) & 0.20 & $317 / 566(56.01)$ \\
\hline anti SRP Ab & $1 / 11(9.1)$ & 20/121 (16.5) & 1.00 & 55 /537 (10.24) \\
\hline anti $H M G C O A$ reductase $A b$ & $1 / 11(9.1)$ & $9 / 28(32.1)$ & 1.00 & $51 / 105$ (48.57) \\
\hline anti-synthetases $A b$ & 2/11 (18.2) & $26 / 120(21.6)$ & 1.00 & $112 / 538(20.82)$ \\
\hline anti-Jo1 Ab & $1 / 11(9.1)$ & $22 / 125(17.6)$ & 0.69 & $99 / 562(17.61)$ \\
\hline anti-PL12 Ab & $0 / 11(0.0)$ & $3 / 120(2.5)$ & 1.00 & $6 / 538(1.11)$ \\
\hline anti-PL7 Ab & $1 / 11(9.0)$ & $1 / 120(0.8)$ & 0.16 & $7 / 538(1.3)$ \\
\hline anti-Ro52 Ab & 4/11 (36.4) & $56 / 125(44.8)$ & 0.75 & $133 / 302(44.04)$ \\
\hline anti-SSA60 Ab & $1 / 11(9.1)$ & $17 / 124$ (13.7) & 1.00 & $44 / 281$ (15.65) \\
\hline
\end{tabular}

